\title{
Eucommia bark (Du-Zhong) improves diabetic nephropathy without altering blood glucose in type I-like diabetic rats
}

This article was published in the following Dove Press journal:

Drug Design, Development and Therapy

2 March 2016

Number of times this article has been viewed

\author{
Ho-Shan Niu' \\ I-Min Liu ${ }^{2}$ \\ Chiang-Shan $\mathrm{Niu}^{\prime}$ \\ Po-Ming $\mathrm{Ku}^{3,4}$ \\ Chao-Tien $\mathrm{Hsu}^{5}$ \\ Juei-Tang Cheng ${ }^{4,6}$ \\ 'Department of Nursing, Tzu Chi \\ University of Science and Technology, \\ Hualien City, ${ }^{2}$ Department of \\ Pharmacy \& Graduate Institute \\ of Pharmaceutical Technology, \\ Tajen University, Pingtung County, \\ ${ }^{3}$ Department of Cardiology, \\ ${ }^{4}$ Department of Medical Research, \\ Chi-Mei Medical Center, Yong Kang, \\ Tainan City, ${ }^{5}$ Department of Pathology, \\ E-DA Hospital, I-Shou University, \\ Kaohsiung City, ${ }^{6}$ Institute of Medical \\ Science, College of Health Science, \\ Chang Jung Christian University, \\ Guei-Ren, Tainan City, Taiwan
}

Background: Eucommia bark, Eucommia ulmoides Oliver barks (Du-Zhong in Mandarin), is an herb used for renal dysfunction in Chinese traditional medicine. In an attempt to develop this herb as a treatment for diabetic nephropathy (DN), we investigated the effects of Du-Zhong on renal dysfunction in type 1-like diabetic rats.

Methods: Streptozotocin (STZ) was used to induce type 1-like diabetes in rats (STZ-diabetic rats). In addition to hyperglycemia, STZ-diabetic rats showed significant nephropathy, including higher plasma levels of blood urea nitrogen, creatinine, and renal fibrosis. Western blot analysis of renal cortical tissue was applied to characterize the changes in potential signals related to nephropathy.

Results: Oral administration of Du-Zhong ( $1 \mathrm{~g} / \mathrm{kg} /$ day) to STZ-diabetic rats for 20 days not only decreased the plasma levels of blood urea nitrogen and creatinine but also improved renal fibrosis, whereas the plasma glucose level was not changed. The higher expressions of protein levels of transforming growth factor-beta (TGF- $\beta$ ) and connective tissue growth factor in diabetic rats were markedly attenuated by Du-Zhong. The increased phosphorylation of Smad2/3 in STZ-diabetic rats was also reduced by Du-Zhong. However, Du-Zhong cannot reverse the hyperglycemia-induced overproduction of signal transducers and activators of transcription 3 in the diabetic kidney.

Conclusion: Oral administration of Du-Zhong improves STZ-induced DN in rats by inhibiting TGF- $\beta /$ Smad signaling and suppressing TGF- $\beta /$ connective tissue growth factor expression. Therefore, active principle from Du-Zhong is suitable to develop as new agent for DN in the future. Keywords: diabetic nephropathy, Du-Zhong, transforming growth factor-beta, Smad2/3, connective tissue growth factor, renal dysfunction

\section{Introduction}

Diabetic nephropathy (DN) is a common complication of diabetes and causes millions of deaths worldwide, and some risk factors are closely related to the development of DN, including hyperglycemia and advanced glycosylation products. ${ }^{1,2}$ Additionally, DN is a progressive kidney disease characterized by glomerular hypertrophy, proteinuria, decreased glomerular filtration, and renal fibrosis with the loss of renal function. ${ }^{3}$ Currently, various treatment regimens and combinations of therapies provide only partial renoprotection. Therefore, it is extremely important to identify novel interventions to halt the progression of DN. ${ }^{4,5}$ Recently, the management of DN has attracted much attention in alternative medicine, and several agents from herbal plants are beneficial to improve DN. ${ }^{6,7}$ Thus, the development of alternative medicine or herbal agent(s) to treat $\mathrm{DN}$ is worthwhile. 
Eucommia ulmoides Oliver (a member of the Eucommiaceae family) is a popular tonic herb in Asia. In Chinese traditional medicine, E. ulmoides, named Du-Zhong in Mandarin, is widely used either as a single herb or in combination with other herbs. ${ }^{8}$ Basically, traditional application of Du-Zhong in Chinese medicine has been documented and the main active constituents, lignans and iridoids, in many compounds have been introduced. ${ }^{9}$ Additionally, Du-Zhong is also a popular folk drink and is used as a functional food reinforcing the muscles and lungs, lowering blood pressure, preventing miscarriages, improving liver and kidney tone, and increasing longevity. ${ }^{10} \mathrm{Du}$-Zhong, prepared from leaves or barks, is commonly used as a tonic to improve detoxification and circulation in liver and kidney. ${ }^{11,12}$ Du-Zhong leaves have been used for the treatment of hepatic lipid accumulation and hepatic damage. ${ }^{11,13}$ Studies have shown that Du-Zhong leaf extracts have potent protective effects in various lipid peroxidation models and reduce oxidative damage of biomolecules. ${ }^{11,14}$

Recently, it was reported that extracts of Du-Zhong barks contain the same components as leaves, which have been the focus of medical research. ${ }^{10,15}$ Du-Zhong protects against cadmium-induced oxidative damage in rat kidneys. ${ }^{16}$ Additionally, lignans extracted from the bark of Du-Zhong were mentioned to inhibit angiotensin II-induced proliferation and extracellular matrix (ECM) biosynthesis in rat mesangial cells. ${ }^{17}$ This action was indicated by upregulating P21, P27, and Bax (but not Bcl-2) expression. ${ }^{18}$ Otherwise, Du-Zhong leaves exhibit antidiabetic activity in several diabetic models. ${ }^{9}$ However, the possibility that Du-Zhong could be beneficial in ameliorating diabetic renal damage has not been previously explored. In the present study, type 1-like diabetic rats induced by streptozotocin (STZ-diabetic rats) were employed as an animal model of DN to screen the effects of Du-Zhong on renal damage and clarify the potential mechanism(s). The main purpose was to provide new insight on an alternative medicine to improve DN.

\section{Materials and methods}

\section{Plant material and extraction}

Du-Zhong was purchased from Jinbaoan Trade Co., Ltd (Zhunan Township, Miaoli County 350, Taiwan) in May 2011, and verified by Professor TY Hong of the Department of Biotechnology, College of Pharmacy and Health Care, Tajen University. The voucher specimen (Lot no TUP21602) was deposited in our laboratory. The $10 \mathrm{~kg}$ seeds were ground into 40-mesh powder and extracted with 95\% ethanol (five volumes of ethanol) in a stainless steel extraction tank for 24 hours at room temperature. This ethanol mixture was filtered in the funnels and centrifuged $\left(4^{\circ} \mathrm{C}, 1,350 \times g, 20\right.$ minutes $)$, a process that was repeated three times. All the precipitates were eliminated, and the ethanol extract portion was collected and then concentrated using a rotary evaporator. Du-Zhong was then evaporated to dryness under reduced pressure for the total elimination of alcohol, followed by lyophilization, yielding $\sim 1,213.6 \mathrm{~g}$ of dry residue (w/w yield: $12.1 \%$ ).

\section{Experimental animals}

The male Wistar rats weighing 250-300 g were obtained from the Animal Center of National Cheng Kung University Medical College. The diet of the animals used for the study was a standard laboratory diet (Harlan Teklad, Madison, WI, USA; Cat No 2018). The number of animals in each group of experiment was eight. STZ-diabetic rats were induced by intravenous injection of STZ (65 mg/ $\mathrm{kg}$ ) according to a previously described method. ${ }^{19}$ Animals were considered to be diabetic if they had a plasma glucose concentration of $320 \mathrm{mg} / \mathrm{dL}$ or greater in addition to polyuria and other diabetic features. All studies were performed 2 weeks after the injection of STZ. All animal procedures were conducted in accordance with the internationally accepted principles for laboratory animal use and care as found in the European Economic Community guidelines (EEC Directive of 1986; $86 / 609 /$ EEC) or the US guidelines (National Institutes of Health publication \#85-23, revised in 1985).

\section{Renal evaluation studies}

In the preliminary experiments, Du-Zhong produced decrease of renal indicators from $0.4 \mathrm{mg} / \mathrm{kg}$ in a dose-dependent manner and $1 \mathrm{~g} / \mathrm{kg}$ was identified as the effective dose. Therefore, STZ-diabetic rats were dosed by oral gavage once per day for 20 days with Du-Zhong doses of $1 \mathrm{~g} / \mathrm{kg}$ in a volume of $1.5 \mathrm{~mL} / \mathrm{kg}$ distilled water. A vehicle-treated group of STZdiabetic rats and normal rats was treated with $1.5 \mathrm{~mL} / \mathrm{kg}$ of distilled water during the same treatment period. Animals received a standard rat diet and drank water freely throughout the experimental period. Du-Zhong treatment was continued, although the plasma glucose of STZ-diabetic rats was lower than $350 \mathrm{mg} / \mathrm{dL}$ during the treatment period. On the evening before blood sample collection, animals were restricted to $3 \mathrm{~g}$ of chow (given at 18:00), which was consumed immediately, and thereafter had access to only water. The animals were transferred to metabolic cages (Shineteh Instruments Co., Ltd, Taipei, Taiwan) for urine collection 24 hours before sacrifice. Urine was collected under a layer of toluene (to inhibit bacteria growth) and stored at $4{ }^{\circ} \mathrm{C}$ until analyzed. 
At the end of the 20-day treatment, rats were sacrificed using an intraperitoneal injection of sodium pentobarbital $(50 \mathrm{mg} / \mathrm{kg})$. The kidneys were dissected and rinsed with cold isotonic saline and weighed. The cortical tissues from the right kidney were stored immediately at $-80^{\circ} \mathrm{C}$ in liquid nitrogen for biochemical determinations and Western blot analyses. Additional kidney tissues were fixed in 10\% neutralized formalin for histological analysis.

\section{Measurements of blood parameters}

The concentration of plasma glucose was measured by the glucose oxidase method using an analyzer (Quik-Lab, Ames; Miles Inc., Elkhart, IN, USA). The levels of blood urea nitrogen (BUN) and creatinine were measured using enzymatic methods (Hoffman-La Roche Ltd., Basel, Switzerland) with an automatic analyzer (Roche).

\section{Analysis of urine parameters}

The 24-hour urine samples collected from each diabetic rat and age-matched control were centrifuged at $2,000 \times g$ for $10 \mathrm{~min}$ utes. Urinary albumin concentrations were measured with the Nephrat II ELISA kit (Exocell Inc., Philadelphia, PA, USA; Cat No NR002). The concentration of creatinine in pooled urine samples was determined using a commercial assay kit (Diagnostic Chemicals Ltd., Charlottetown, Canada; Cat No 221-30). All analyses were performed in accordance with the manufacturer's instructions. Creatinine clearance was calculated in individual rats using the relationship: creatinine clearance $=$ urine creatinine $\times($ urine volume $/$ plasma creatinine $) \times$ time .

\section{Western blot analysis}

The kidney tissue was homogenized in $1 \mathrm{~mL}$ of ice-cold hypotonic buffer A (10 mmol/L 4-(2-hydroxyethyl)-1piperazineethanesulfonic acid [pH 7.8], $10 \mathrm{mmol} / \mathrm{L} \mathrm{KCl}$, $2 \mathrm{mmol} / \mathrm{L} \mathrm{MgCl}_{2}, 1 \mathrm{mmol} / \mathrm{L}$ dithiothreitol, $0.1 \mathrm{mmol} / \mathrm{L}$ ethylenediaminetetraacetic acid, $0.1 \mathrm{mmol} / \mathrm{L}$ phenylmethylsulfonylfluoride). A solution of $80 \mu \mathrm{L}$ of $10 \%$ Nonidet P-40 was added to the homogenates, and the mixture was centrifuged for 2 minutes at $14,000 \times g$. Before immunoblotting, the protein concentration of each tissue was determined using a Bio-Rad protein assay kit (Bio-Rad Laboratories Inc., Hercules, CA, USA) and bovine serum albumin as a standard, to ensure equal loading among lanes. Homogenates $(50 \mu \mathrm{g}$ total protein) were separated on a $7.5 \%-15 \%$ polyacrylamide gel and electrophoretically transferred to nitrocellulose membranes. Membranes were blocked with 5\% nonfat dry milk in Tris-buffered saline Tween $(20 \mathrm{mmol} / \mathrm{L}$ Tris, $\mathrm{pH}$ 7.6, $137 \mathrm{mmol} / \mathrm{L} \mathrm{NaCl}$, and $0.1 \%$ Tween 20) for 3 hours at room temperature, followed by an overnight incubation at $4{ }^{\circ} \mathrm{C}$ with polyclonal antibodies against rat transforming growth factor-beta 1 (TGF- $\beta 1$ ) (Abcam plc, Cambridge, UK; Cat No ab66043), connective tissue growth factor (CTGF; Abcam plc; Cat No ab6992), signal transducers and activators of transcription 3 (STAT3; Abcam plc; Cat No ab50761), phosphorylated (p-Smad2/3) antibody (Ser 423/425) (Santa Cruz Biotechnology Inc., Dallas, TX, USA; Cat No sc-11769), or actin (Santa Cruz Biotechnology Inc.; Cat No sc-1616). All antibodies were used at a dilution of 1:1,000. After three times washes with Tris-buffered saline Tween 20, incubation with appropriate horseradish peroxidase-conjugated secondary antibodies was performed for 1 hour at room temperature. After three additional Tris-buffered saline Tween 20 washes, the immunoreactive bands were visualized by enhanced chemiluminescence (Amersham Biosciences, Buckinghamshire, UK) according to the manufacturer's instructions. After comparing with the marker for specificity, the immunoblots of TGF- $\beta 1$ (13 kDa), CTGF (38 kDa), p-Smad2/3 (58 kDa), STAT3 $(88 \mathrm{kDa})$, and $\beta$-actin ( $43 \mathrm{kDa})$ were determined using ATTO Densitograph Software (ATTO Corporation, Tokyo, Japan) and quantified as the ratio to actin.

\section{Histological analysis}

The kidney tissues were removed from each group of rats and fixed in $10 \%$ formaldehyde at $4{ }^{\circ} \mathrm{C}$ for 2 days. Fixed specimens were dehydrated and embedded in paraffin. The specimens were then cut into $5 \mu \mathrm{m}$ thick sections at $50 \mu \mathrm{m}$ intervals and stained with Masson's trichrome stain and hematoxylin and eosin (Muto Pure Chemicals, Tokyo, Japan). All sections were identified under the light microscope by a pathology expert.

\section{Statistical analysis}

The results are presented as the mean \pm standard deviation for each group of animals at the number (n) indicated. Differences among groups were analyzed by one-way analysis of variance. Dunnett range post hoc comparisons were used to determine the source of significant differences as appropriate. A $P$-value of 0.05 or less was considered statistically significant.

\section{Results \\ Effects of Du-Zhong on renal function parameters and plasma glucose levels in STZ-diabetic rats}

The plasma levels of BUN and creatinine in STZ-diabetic rats were markedly higher than those in normal rats. 
Table I Effects of Du-Zhong on renal function parameters and plasma glucose levels in STZ-diabetic rats

\begin{tabular}{|c|c|c|c|}
\hline \multirow[t]{2}{*}{ Parameters } & \multirow{2}{*}{$\frac{\text { Normal rats }}{\text { Vehicle }}$} & \multicolumn{2}{|c|}{ STZ-diabetic rats } \\
\hline & & Vehicle & Du-Zhong \\
\hline Plasma glucose (mg/dL) & $103.35 \pm 2.46^{\# \#}$ & $401.72 \pm 10.64 * *$ & $409.53 \pm 16.37 * *$ \\
\hline BUN (mg/dL) & $|4.88 \pm 0.2|^{\#}$ & $28.00 \pm 0.95 * *$ & $17.63 \pm 0.75 * * \ldots$ \\
\hline Plasma creatinine (mg/dL) & $0.44 \pm 0.02^{\#}$ & $0.54 \pm 0.02 *$ & $0.40 \pm 0.03^{\#}$ \\
\hline Creatinine clearance $(\mathrm{mL} / \mathrm{min})$ & $4.18 \pm 0.62^{\#}$ & $1.93 \pm 0.57 * *$ & $3.11 \pm 0.72 * \ldots$ \\
\hline 24-hour urine volume (mL/day) & $8.92 \pm 1.73^{\#}$ & $29.62 \pm 2.98 * *$ & $20.16 \pm 3.14^{* \ldots}$ \\
\hline 24-hour urine protein (mg/day) & $6.81 \pm 3.26 \#$ & $27.24 \pm 3.7 \mathrm{I} * *$ & $18.47 \pm 4.82^{*}$ \\
\hline
\end{tabular}

Notes: Du-Zhong $(\mathrm{g} / \mathrm{kg} / \mathrm{day})$ was orally administered into STZ-diabetic rats for 20 days. The values represent the mean \pm standard deviation of eight animals. $* P<0.05$, $* * P<0.01$ compared with the control (Wistar rats). ${ }^{\#}<0.05$ and ${ }^{\# P} P<0.01$ compared with STZ-diabetic rats (vehicle).

Abbreviations: BUN, blood urea nitrogen; STZ, streptozotocin.

Both BUN and creatinine were decreased in STZ-diabetic rats receiving oral intake of Du-Zhong (1 $\mathrm{g} / \mathrm{kg} /$ day) for 20 days (Table 1). Additionally, STZ-diabetic rats showed an increase in 24-hour urine volume, accompanied by an increase in urine protein excretion (Table 1). After 20 days of Du-Zhong ( $1 \mathrm{~g} / \mathrm{kg} /$ day) treatment, 24-hour urine volume and 24-hour urine protein excretion in STZ-diabetic rats were markedly less than those of their vehicle-treated counterparts (Table 1).

A significant increase in fasting blood glucose in STZdiabetic rats was observed compared with the control group.
However, Du-Zhong did not affect the STZ-induced hyperglycemia (Table 1).

\section{Effects of Du-Zhong on the renal histology in STZ-diabetic rats}

Histological assessment in renal samples revealed that DuZhong ameliorated the pathologic changes associated with diabetes, including glomerular hypertrophy, mesangial expansion, and tubular atrophy/dilation (Figure 1A). Furthermore, the analysis of collagen distribution by Masson's trichrome stain indicated that Du-Zhong administration

A

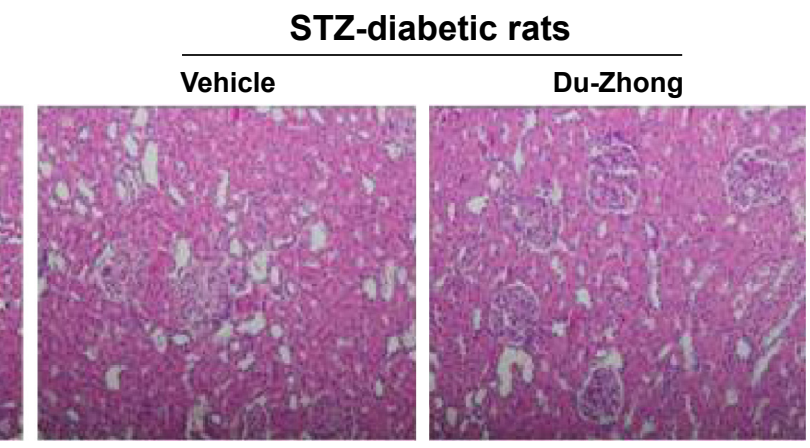

B

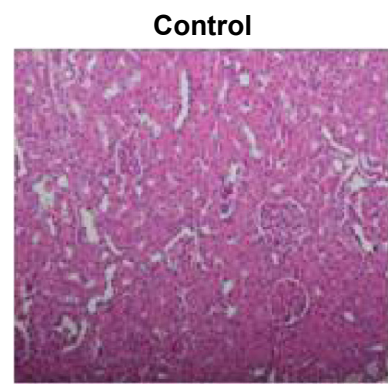

STZ-diabetic rats
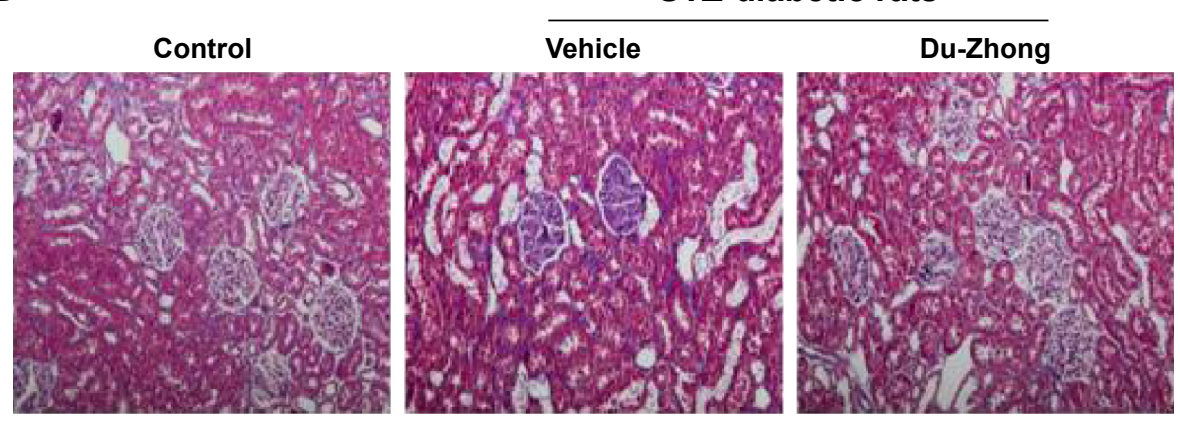

Figure I Histological changes influenced by Du-Zhong.

Notes: Representative photomicrographs of hematoxylin and eosin (A) and Masson's trichrome stained (B) kidney sections from rats treated with or without Du-Zhong. STZ-diabetic rats were dosed by oral gavage once daily for 20 days with I g/kg Du-Zhong (Du-Zhong). Normal (control) or STZ-diabetic rats (vehicle) were administered the same volume of vehicle used to prepare the test compounds. The photomicrographs were taken at a magnification of $\times 200$.

Abbreviation: STZ, streptozotocin. 
A
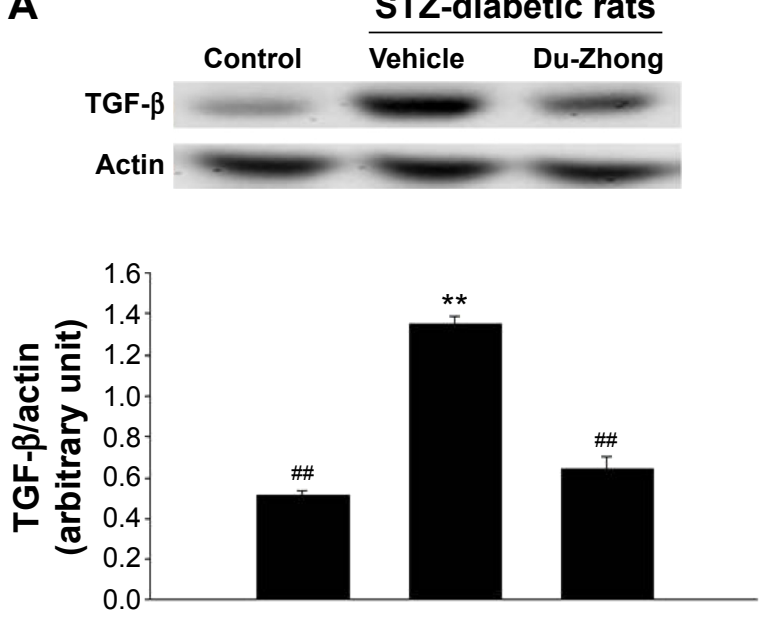

B
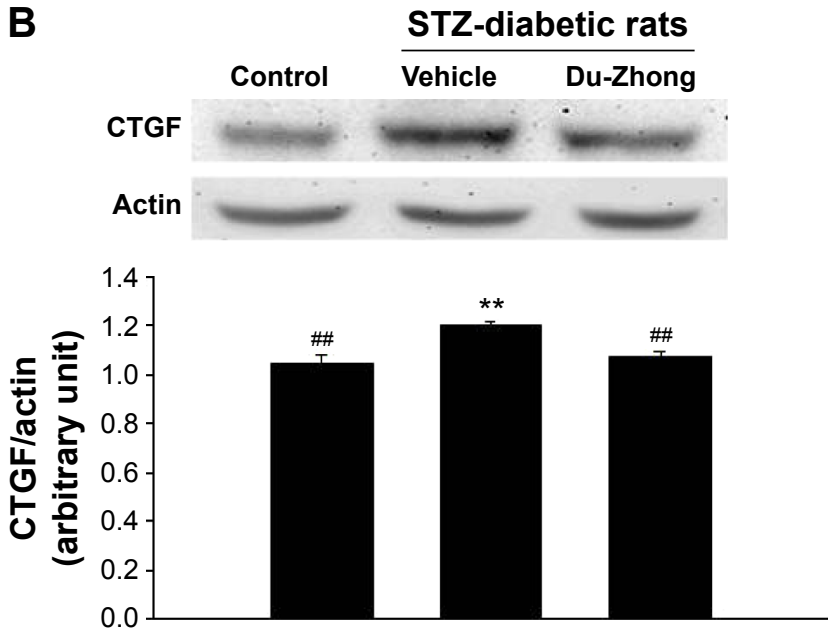

Figure 2 Effects of Du-Zhong on protein expression of TGF-beta and CTGF in renal tissues of rats.

Notes: Representative immunoblots of protein expression of TGF- $\beta$ (A) and CTGF (B) in renal tissues of rats. STZ-diabetic rats were dosed by oral gavage once daily for 20 days with I g/kg Du-Zhong (Du-Zhong). Normal (control) or STZ-diabetic rats received the vehicle treatment (vehicle) at the same volume of vehicle that was used to prepare the test solution. Ratio of TGF- $\beta$ /actin or CTGF/actin is expressed as the mean \pm standard deviation ( $n=4$ per group) in each column. ** $P<0.01$ compared with data obtained from vehicle-treated normal rats. ${ }^{\# P} P 0.01$ compared with data obtained from vehicle-treated STZ-diabetic rats.

Abbreviations: CTGF, connective tissue growth factor; STZ, streptozotocin; TGF- $\beta$, transforming growth factor-beta.

normalized the intraglomerular and tubulointerstitial fibrosis in diabetic rats (Figure 1B).

\section{Effects of Du-Zhong on TGF- $\beta$ and CTGF protein levels in the kidneys of STZ- diabetic rats}

The renal protein levels of TGF- $\beta$ and CTGF in STZdiabetic rats were significantly higher (1.4 and 1.3 times, respectively) compared with those of their vehicle-treated controls (Figure 2). After 20 days of Du-Zhong (1 g/kg/ day) treatment, the higher renal levels of TGF- $\beta$ and CTGF were $-57.3 \%$ and $20.3 \%$ lower in the STZ-diabetic rats compared with the levels in their vehicle-treated counterparts (Figure 2).

\section{Effects of Du-Zhong on phosphorylation degree of Smad2/3 and STAT3 protein levels in the kidneys of STZ-diabetic rats}

STZ treatment significantly increased the phosphorylation degree of Smad2 (1.5-fold increase relative to that of vehicle-treated rats) in the kidney (Figure 3). These STZinduced upregulations in phosphorylation degree of Smad2/3 were reversed in the kidney after treatment with Du-Zhong $(51.2 \% \pm 4.7 \%$ decrease relative to those in vehicle-treated STZ-diabetic rats) (Figure 3).

Otherwise, STAT3 protein levels in the diabetic rats were markedly raised about 3.5 times of that in normal rats. Treatment with Du-Zhong at $1 \mathrm{~g} / \mathrm{kg} /$ day for 20 days decreased renal STAT3 protein only by $9.3 \%$ (Figure 4 ).

\section{Discussion}

In the present study, we employed STZ-diabetic rats to investigate the effects of Du-Zhong on DN. This animal model mimicked type 1 diabetes due to insulin deficiency and was also used to evaluate DN. ${ }^{20}$ It is widely known that plasma BUN and creatinine levels are the reliable indicators

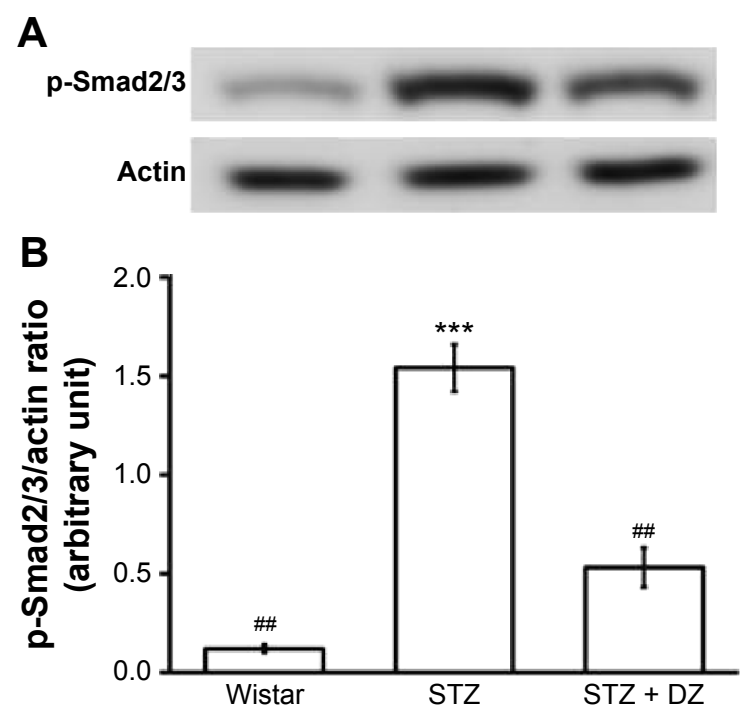

Figure 3 Effect of Du-Zhong on phosphorylated Smad 2/3 level in renal tissues of rats.

Notes: (A) Effects of treatments on p-Smad2/3 in renal tissues of rats. (B) STZ-diabetic rats were dosed by oral gavage once daily for 20 days with I g/kg Du-Zhong (STZ + DZ). Normal or STZ-diabetic rats received the vehicle treatment at the same volume of vehicle used to prepare the test solution. Ratio of $\mathrm{p}$-Smad2/3/actin is expressed as the mean \pm standard deviation ( $n=4$ per group) in each column. $* * * p<0.001$ compared with data obtained from vehicle-treated normal Wistar rats (Wistar). $P<0.01$ compared with data obtained from vehicle-treated STZ-diabetic rats (STZ).

Abbreviations: DZ, Du-Zhong; p-Smad2/3, phosphorylated Smad2/3; STZ, streptozotocin. 


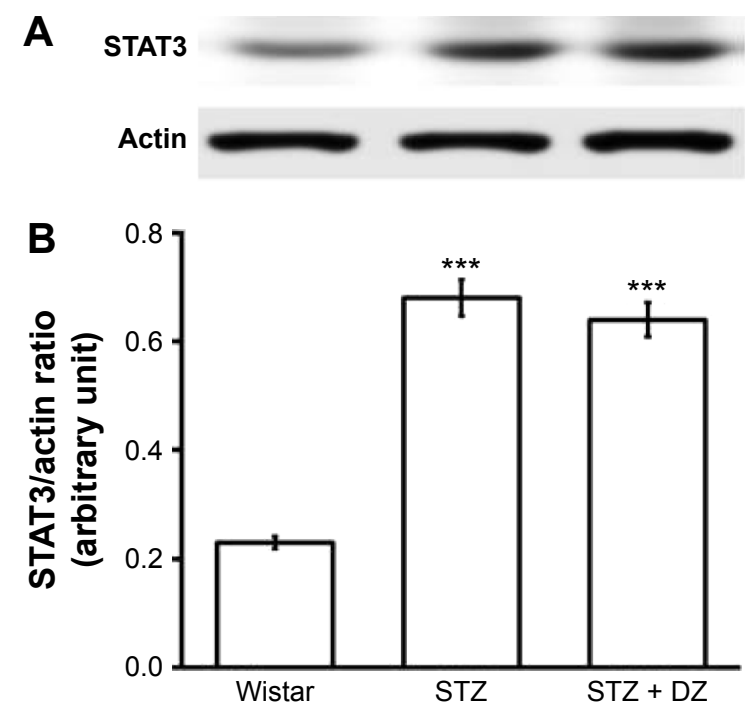

Figure 4 Effect of Du-Zhong on protein expression of STAT3 in renal tissues of rats.

Notes: (A) Representative immunoblots of protein expression of STAT3 in renal tissues of rats. (B) STZ-diabetic rats were dosed by oral gavage once daily for 20 days with I g/kg Du-Zhong (STZ + DZ). Normal or STZ-diabetic rats were administered the same volume of vehicle used to prepare the test solution. Ratio of STAT3 over actin is expressed as the mean \pm standard deviation ( $n=4$ per group) in each column. $* * * P<0.001$ compared with data obtained from vehicle-treated normal rats (Wistar). Abbreviations: DZ, Du-Zhong; STAT3, signal transducers and activators of transcription 3; STZ, streptozotocin.

to assess renal function. ${ }^{21}$ In the present study, untreated STZ-induced diabetic rats exhibited significant increases in plasma BUN and creatinine levels. Du-Zhong significantly decreased the plasma BUN and creatinine levels in STZdiabetic rats. STZ-diabetic rats treated with Du-Zhong also showed an impressive decrease in the amount of proteinuria in parallel with the decrease in urinary volume. The kidneyprotective effects of Du-Zhong were further confirmed by the finding that this treatment attenuated the structural abnormalities of DN. Thus, we demonstrated that treatment with Du-Zhong attenuated DN and the loss of renal function in STZ-diabetic rats.

Recent studies have demonstrated that early renal hypertrophy is detrimental to the kidney in the long term and is a precursor of development of renal fibrosis. ${ }^{22}$ Histological analysis by Masson's trichrome stain also showed that there was a marked decrease in the kidney fibrosis in STZ-diabetic rats treated with Du-Zhong. Our present study demonstrated that Du-Zhong not only attenuated the structural abnormalities of DN but also inhibited renal fibrosis in the diabetes-induced expression of ECM. Overall, the beneficial effects of Du-Zhong on STZ-induced renal damage were identified.

Although the hypoglycemic effect of $E$. ulmoides leaves in STZ-diabetic rats has been demonstrated, ${ }^{23}$ the bark of Du-Zhong used in the present study had a limited effect on hyperglycemia induced by STZ. This finding suggested that the improvement in renal function due to Du-Zhong seems unrelated to decreases in hyperglycemia. Although the exact mechanisms of renal hypertrophy are still unclear, several growth factors, cytokines, and chemokines have been implicated in the development of renal hypertrophy. ${ }^{24,25}$ Previous findings suggest that the Janus kinase/STAT pathway, especially the Janus kinase 2/STAT1/STAT3dependent axis, contributes to high glucose-mediated renal cell responses, including enhanced expression of genes involved in leukocyte infiltration, cell growth, and fibrosis. ${ }^{26}$ Therefore, we determined the changes in STAT3 expression but not the phosphorylated STAT3 in this study. However, as shown in Figure 4, Du-Zhong did not reverse the overproduction of STAT3 in the diabetic kidney. These results suggest that the renal protective effects of Du-Zhong may not be related to the suppression of hyperglycemia-induced activation of STAT proteins. It proves that the beneficial effect of Du-Zhong in rats with DN is not mediated by its antihyperglycemic activity.

TGF- $\beta$ is an effector molecule that has been extensively studied as a major mediator of the hypertrophic and prosclerotic changes in diabetic kidney disease. ${ }^{27}$ Additionally, CTGF is associated with the pathogenesis of DN because the inhibition of CTGF expression improved renal fibrosis. ${ }^{28}$ CTGF can cooperate with TGF- $\beta$ to induce sustained fibrosis and to exacerbate ECM production. ${ }^{29}$ After treatment with Du-Zhong, overexpression of TGF- $\beta 1$ or CTGF in the kidney of STZ-diabetic rats was markedly reduced. This result suggests that Du-Zhong may improve DN by the suppression of TGF- $\beta /$ CTGF expressions. However, the mechanism(s) for Du-Zhong-induced reduction of overexpressed TGF- $\beta 1$ shall be investigated in the future.

Members of the TGF- $\beta$ superfamily transduce intracellular signals by Smad proteins; Smad 2 and $\mathrm{Smad} 3$ act in the TGF- $\beta$ /activin pathway. Smad2/3 form heteromeric complexes with Smad4 and translocate into the nucleus to regulate the transcription of target genes. A study demonstrated that TGF- $\beta$ signals mediate the renal fibrosis through Smad2/3. ${ }^{27}$ To confirm the effect of Du-Zhong on the glomerular TGF- $\beta$ expression in diabetic rats, we detected the phosphorylation of Smad2/3 using Western blot. Basically, phosphorylation is compared using $\mathrm{p}-\mathrm{Smad} 2 / 3$ over Smad2/3. Because Smad2/3 was not changed in regular, we applied actin as an alternative way. In the present study, STZ-diabetic rats revealed an increase in phosphorylation of $\mathrm{Smad} 2 / 3$ that was attenuated by Du-Zhong. Thus, the renal protective effect of Du-Zhong may be partly through the inhibition of 
the TGF- $\beta$ /Smad signaling pathway. Although the signaling pathway is established in renal pathology, merit of Du-Zhong in DN was not mentioned in animals before.

According to previous studies, some active components in the barks of Du-Zhong have been identified, including iridoids, flavonoids, and phenolic compounds. ${ }^{10,15}$ Recently, 112 compounds have been isolated, including 28 lignans, 24 iridoids, 27 phenolics, six steroids, five terpenoids, 13 flavonoids, and nine other compounds. ${ }^{9}$ Officially, pinoresinol-4,4'-di- $O$ - $\beta$-D-glucopyranoside is used as the quality control marker for Du-Zhong bark. Chlorogenic acid and some iridoids, aucubin, geniposidic acid, and geniposide, have been demonstrated to be the main and active ingredients in this plant. ${ }^{9}$ However, the active principle(s) for effectiveness of Du-Zhong remained obscure. Similar to it, the potential mechanism(s) for Du-Zhong-induced improvement of DN should be investigated in the future.

Taken together, our results suggest that Du-Zhong has beneficial effects on DN that are mainly mediated by the attenuation of TGF- $\beta$ and CTGF in kidney to improve renal dysfunction. Additionally, Du-Zhong reduces harmful Smad-mediated cell responses in the diabetic kidney, thus indicating the potential ability of Du-Zhong to halt the progression of DN. Therefore, Du-Zhong and/or the contained active principle(s) can be developed as an alternative agent to improve $\mathrm{DN}$ in the future.

\section{Acknowledgments}

The authors would like to thank Dr LJ Chen and Mr YC Chen for their skillful technical assistance. The present study was supported in part by a grant from Tzu Chi University of Science and Technology (TCCT-1022A07), Hualien City, Taiwan. H-S Niu and C-S Niu are brothers in same family.

\section{Disclosure}

The authors declare no conflicts of interest in this work.

\section{References}

1. Sun YM, Su Y, Li J, Wang LF. Recent advances in understanding the biochemical and molecular mechanism of diabetic nephropathy. Biochem Biophys Res Commun. 2013;433(4):359-361.

2. Arora MK, Singh UK. Molecular mechanisms in the pathogenesis of diabetic nephropathy: an update. Vascul Pharmacol. 2013;58(4):259-271.

3. Ziyadeh FN, Wolf G. Pathogenesis of the podocytopathy and proteinuria in diabetic glomerulopathy. Curr Diabetes Rev. 2008;4(1):39-45.

4. Turgut F, Bolton WK. Potential new therapeutic agents for diabetic kidney disease. Am J Kidney Dis. 2010;55(5):928-940.

5. Oluwole Busayo A, Laura Z, Olufunke Olubusola D, Oluwafunmike Sharon A, Luciana D, Ezekiel Ademola CM. Ameliorative effects of ethanolic leaf extract of Azadirachta indica on renal histologic alterations in streptozotocin-induced diabetic rats. Am J Chin Med. 2011;39(5): 903-916.
6. Chennasamudram SP, Kudugunti S, Boreddy PR, Moridani MY, Vasylyeva TL. Renoprotective effects of (+)-catechin in streptozotocininduced diabetic rat model. Nutr Res. 2012;32(5):347-356.

7. Yang QH, Liang Y, Xu Q, Zhang Y, Xiao L, Si LY. Protective effect of tetramethylpyrazine isolated from Ligusticum chuanxiong on nephropathy in rats with streptozotocin-induced diabetes. Phytomedicine. 2011;18(13):1148-1152.

8. Du Y, Wu Y, Cao X, et al. Inhibition of mammalian thioredoxin reductase by black tea and its constituents: implications for anticancer actions. Biochimie. 2009;91(3):434-444.

9. He X, Wang J, Li M, et al. Eucommia ulmoides Oliv.: ethnopharmacology, phytochemistry and pharmacology of an important traditional Chinese medicine. J Ethnopharmacol. 2014;151(1):78-92.

10. Yen GC, Hsieh CL. Reactive oxygen species scavenging activity of Du-zhong (Eucommia ulmoides Oliv.) and its active compounds. J Agric Food Chem. 2000;48(8):3431-3436.

11. Hung MY, Fu TY, Shih PH, Lee CP, Yen GC. Du-Zhong (Eucommia ulmoides Oliv.) leaves inhibits CCl4-induced hepatic damage in rats. Food Chem Toxicol. 2006;44(8):1424-1431.

12. Li L, Yan J, Hu K, et al. Protective effects of Eucommia lignans against hypertensive renal injury by inhibiting expression of aldose reductase. J Ethnopharmacol. 2012;139(2):454-461.

13. Choi MS, Jung UJ, Kim HJ, et al. Du-zhong (Eucommia ulmoides Oliver) leaf extract mediates hypolipidemic action in hamsters fed a high-fat diet. Am J Chin Med. 2008;36(1):81-93.

14. Luo J, Tian C, Xu J, Sun Y. Studies on the antioxidant activity and phenolic compounds of enzyme-assisted water extracts from Duzhong (Eucommia ulmoides Oliv.) leaves. J Enzyme Inhib Med Chem. 2009;24(6):1280-1287.

15. Guan S, Su W. Advances in the study of chemical constituents and pharmacology of Eucommia ulmoides Oliver. Zhong Yao Cai. 2003;26(2): $124-129$.

16. Liu E, Han L, Wang J, et al. Eucommia ulmoides bark protects against renal injury in cadmium-challenged rats. J Med Food. 2012;15(3): 307-314.

17. Li ZY, Deng XL, Huang WH, et al. Lignans from the bark of Eucommia ulmoides inhibited Ang II-stimulated extracellular matrix biosynthesis in mesangial cells. Chin Med. 2014;9(1):8.

18. Jing X, Huang WH, Tang YJ, et al. Eucommia ulmoides Oliv. (Du-Zhong) lignans inhibit angiotensin ii-stimulated proliferation by affecting P21, P27, and Bax expression in rat mesangial cells. Evid Based Complement Alternat Med. 2015;2015:987973.

19. Liu IM, Chen WC, Cheng JT. Mediation of beta-endorphin by isoferulic acid to lower plasma glucose in streptozotocin-induced diabetic rats. J Pharmacol Exp Ther. 2003;307(3):1196-1204.

20. Luippold G, Klein T, Mark M, Grempler R. Empagliflozin, a novel potent and selective SGLT-2 inhibitor, improves glycaemic control alone and in combination with insulin in streptozotocin-induced diabetic rats, a model of type 1 diabetes mellitus. Diabetes Obes Metab. 2012;14(7):601-607.

21. Ozcan F, Ozmen A, Akkaya B, Aliciguzel Y, Aslan M. Beneficial effect of myricetin on renal functions in streptozotocin-induced diabetes. Clin Exp Med. 2012;12(4):265-272.

22. Pohlers D, Brenmoehl J, Loffler I, et al. TGF-beta and fibrosis in different organs - molecular pathway imprints. Biochim Biophys Acta. 2009;1792(8):746-756.

23. Lee MK, Kim MJ, Cho SY, et al. Hypoglycemic effect of Du-zhong (Eucommia ulmoides Oliv.) leaves in streptozotocin-induced diabetic rats. Diabetes Res Clin Pract. 2005;67(1):22-28.

24. Navarro-Gonzalez JF, Mora-Fernandez C. The role of inflammatory cytokines in diabetic nephropathy. J Am Soc Nephrol. 2008;19(3): 433-442.

25. Fornoni A, Ijaz A, Tejada T, Lenz O. Role of inflammation in diabetic nephropathy. Curr Diabetes Rev. 2008;4(1):10-17.

26. Marrero MB, Banes-Berceli AK, Stern DM, Eaton DC. Role of the JAK/STAT signaling pathway in diabetic nephropathy. Am J Physiol Renal Physiol. 2006;290(4):F762-F768. 
27. Lan HY. Transforming growth factor-beta/Smad signalling in diabetic nephropathy. Clin Exp Pharmacol Physiol. 2012;39(8):731-738.

28. Brigstock DR. Connective tissue growth factor (CCN2, CTGF) and organ fibrosis: lessons from transgenic animals. J Cell Commun Signal. 2010;4(1):1-4.
29. Sonnylal S, Shi-Wen X, Leoni P, et al. Selective expression of connective tissue growth factor in fibroblasts in vivo promotes systemic tissue fibrosis. Arthritis Rheum. 2010;62(5):1523-1532.

\section{Publish your work in this journal}

Drug Design, Development and Therapy is an international, peerreviewed open-access journal that spans the spectrum of drug design and development through to clinical applications. Clinical outcomes, patient safety, and programs for the development and effective, safe, and sustained use of medicines are a feature of the journal, which has also been accepted for indexing on PubMed Central. The manuscript management system is completely online and includes a very quick and fair peer-review system, which is all easy to use. Visit http://www.dovepress.com/testimonials.php to read real quotes from published authors.

Submit your manuscript here: http://www.dovepress.com/drug-design-development-and-therapy-journal 